\title{
Peripherally inserted central catheter procedure at the bedside by a nephrologist is safe and successful
}

\author{
Seong Cho \\ Division of Nephrology, Department of Internal Medicine, Samsung Changwon Hospital, Sungkyunkwan University School of Medicine, \\ Changwon, Republic of Korea
}

Background: Peripherally inserted central catheter (PICC) use among critically ill patients with or without acute kidney injury (AKI) has gradually increased. Ultrasound-guided bedside PICC insertion in intensive care units (ICU) has been reported to be safe and effective. Reports of PICC insertion by a nephrologist without fluoroscopy, however, are relatively rare.

Methods: This retrospective study included patients $(n=224)$ who had a PICC inserted by a single nephrologist at Samsung Changwon Hospital from January 2019 to June 2020. Group 1 patients ( $n=98$ ) had PICCs inserted under ultrasound guidance, while group 2 patients $(n=126)$ had PICCs inserted under fluoroscopic guidance. Success rates, multiple puncture rates, and malposition rates were compared between the two groups.

Results: Underlying comorbidities (sepsis, AKI, ventilator use, and shock) were more common in group 1 than in group 2 . Success rates were comparable between the two groups (93.9\% vs. $97.6 \%, p=0.171)$. Multiple puncture rate among successful cases $(4.1 \%$ vs. $0.0 \%, p=0.035$ ) was higher in group 1 than group 2 . Excluding central vein occlusion cases, malposition occurred only one in group 1.

Conclusion: Bedside PICC insertion by a nephrologist is easy and safe to perform in comorbid patients who are difficult to move to the angiography room. The success rate of ultrasound-guided PICC insertions was comparable to that of PICC insertion performed under fluoroscopic guidance. In the life-threatening ICU setting, PICCs can be successfully placed by the interventional nephrologists.

Keywords: Peripheral catheterization, Critical illness, Intensive care unit, Peripherally inserted central catheter

\section{Introduction}

A peripherally inserted central catheter (PICC) is essential for prolonged chemotherapy, nutritional support, and antibiotic injections [1,2]. The number of elderly and longterm comorbid patients who require PICC insertion by a nephrologist to achieve adequate blood access is gradually increasing [3]. PICC insertion is commonly conducted with fluoroscopic procedures in the angiography room. However, patients with poor medical conditions such as ventilator dependency

Received: July 6, 2020; Revised: October 5, 2020; Accepted: October 13, 2020

Editor: Jeonghwan Lee, Seoul National University, Seoul, Republic of Korea

Correspondence: Seong Cho

Division of Nephrology, Department of Internal Medicine, Samsung Changwon Hospital, Sungkyunkwan University School of Medicine, 158 Paryong-ro, Masanhoewon-gu, Changwon 51353, Republic of Korea. E-mail: chaecho67@gmail.com

ORCID: https://orcid.org/0000-0003-3892-8376

Copyright (C) 2021 The Korean Society of Nephrology

(a) This is an Open Access article distributed under the terms of the Creative Commons Attribution Non-Commercial and No Derivatives License (http:// creativecommons.org/licenses/by-nc-nd/4.0/) which permits unrestricted non-commercial use, distribution of the material without any modifications, and reproduction in any medium, provided the original works properly cited. 
or shock and patients who were required continuous renal replacement therapy (CRRT) or extracorporeal membrane oxygenation (ECMO) were needed to perform PICC insertion at bedside [4-6]. Lack of angiography room availability may also result in the need for bedside PICC insertion. Compared to fluoroscopy-guided insertion, bedside insertion using ultrasound guidance has several disadvantages such as dislocation of the catheter tip to the jugular vein or other inadequate locations and lack of adequate visualization to determine the cause of guidewire passage failures such as vein stenosis or kinking. Nevertheless, many intensivists have reported high bedside PICC insertion success rates [4-6]. However, reports comparing PICC bedside insertion to fluoroscopic insertion success rates and nephrologist experiences within a single practice are rare. Therefore, this study compared success rates between bedside PICC insertion and insertion conducted under fluoroscopic guidance by a single nephrologist.

\section{Methods}

This study retrospectively analyzed patients who had a PICC inserted by a nephrologist of Department of Nephrology, Samsung Changwon Hospital in Changwon, Korea from January 2019 to June 2020. In general, PICC insertion was conducted in the angiography room; these patients were assigned to group 2. PICC insertion among patients who were difficult to transfer to the angiography room due to comorbidities was performed at the bedside and these patients were classified as group 1 patients. Patient characteristics including age, sex, serum creatinine, estimated glomerular filtration rate (eGFR), septic condition, shock state, presence of artificial respiration, severe heart failure, acute kidney injury (AKI), postoperative surgery, and gastrointestinal bleeding were considered. Information about the side of the body where the PICC was inserted was recorded. PICC insertion was considered appropriate only when the scheduled duration of use was longer than 6 days or non-peripherally compatible infusions were needed. In patients with chronic kidney disease (CKD) stage $3 \mathrm{~b}$ to 5 , possible access creation needs were considered and PICC insertion was avoided if possible; therefore, these patients were excluded in this study.

The patient was placed in a supine position by abduction, and, when determining the arm to be treated, the side without any suspicion of proximal vein stenosis (previous catheter insertion site) was considered first. To prevent blood infection, the procedure was performed using sterile methods. A 5-French (Fr) dual-lumen catheter was used, and $2 \%$ chlorhexidine was used to prevent catheter-related infections. In both methods, an appropriate vein (more superficial, larger, or more distant from an artery) was selected using ultrasound (Supplementary Fig. 1, available online). Then, a 0.018-inch guidewire was inserted after puncture of the vein using a micro-puncture needle (Fig. $1 \mathrm{~A}-\mathrm{C})$. After inserting the 5-Fr sheath in group 2 patients, a long guidewire was introduced, and the guidewire tip was positioned between the superior vena cava (SVC) and midportion of the right atrium (RA). Next, the operator measured the length from the sheath to a $60-\mathrm{cm}$ marker at the guidewire surface, and catheter length was calculated as $60 \mathrm{~cm}$ minus the measured length. The operator trimmed the catheter to the desired length and inserted the catheter over the guidewire to a location between the SVC and midportion of the RA (Fig. 2). Group 1 patients had a 5-Fr sheath introduced using ultrasound as in group 2 patients (Fig. 1D). After sheath insertion, the operator calculated catheter length by summing the straight-line distance from the sheath insertion site to the mid-clavicular line (Fig. 1E) and the straight-line distance from the mid-clavicular line to the third intercostal space in the right parasternal area (Fig. 1F) [7]. The operator trimmed catheters according to the calculated length (Fig. $1 \mathrm{G}$ ). While passing through the guidewire, a neck sonogram (Supplementary Fig. 2 and 3, available online) was used to observe the internal jugular vein to confirm that the guidewire had not been misdirected to the neck. When the guidewire could be advanced without resistance, a trimmed catheter was inserted along the guidewire (Fig. 1H). After the procedure, the location of the catheter was confirmed by simple chest anteroposterior imaging. Heparin flush, 100 units $/ \mathrm{mL}$, was injected as a single dose into the PICC line using a volume of solution equivalent to that of the indwelling catheter for thrombosis prevention.

Criteria for success were based on functional status (good inflow/outflow) and chest posteroanterior findings (catheter tip located between SVC and midportion of the RA; Fig. 2). Failure refers to functional status (substandard inflow/outflow) or catheter tip malposition. Failure can be due to one of three issues: 1) puncture failure, 2) catheter or 

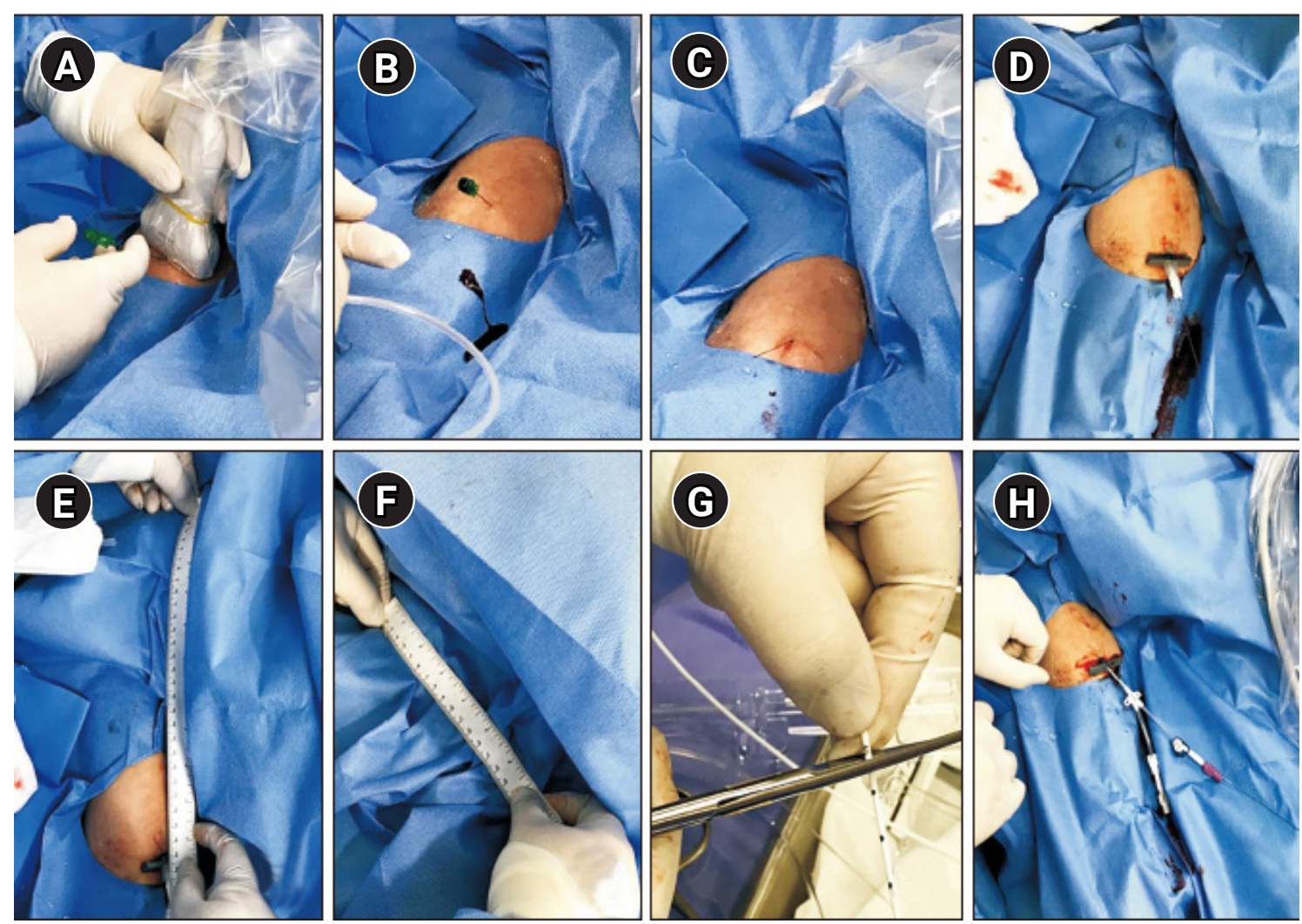

Figure 1. Implementation process. (A-C) The patient was fixed in a supine position by abduction, an appropriate vein was selected using ultrasound, and then a 0.018-inch guidewire was inserted after puncture of the vein using a micro-puncture needle. (D) A 5-French sheath was introduced. (E) After sheath insertion, catheter length was calculated by summing the straight-line distance from the sheath insertion site to the mid-clavicular line and $(F)$ the straight-line distance from the mid-clavicular line to the third intercostal space in the right parasternal area. $(G)$ The operator trimmed catheters according to calculated length. $(H)$ When the guidewire proceeded without resistance, a trimmed catheter was inserted along the guidewire.

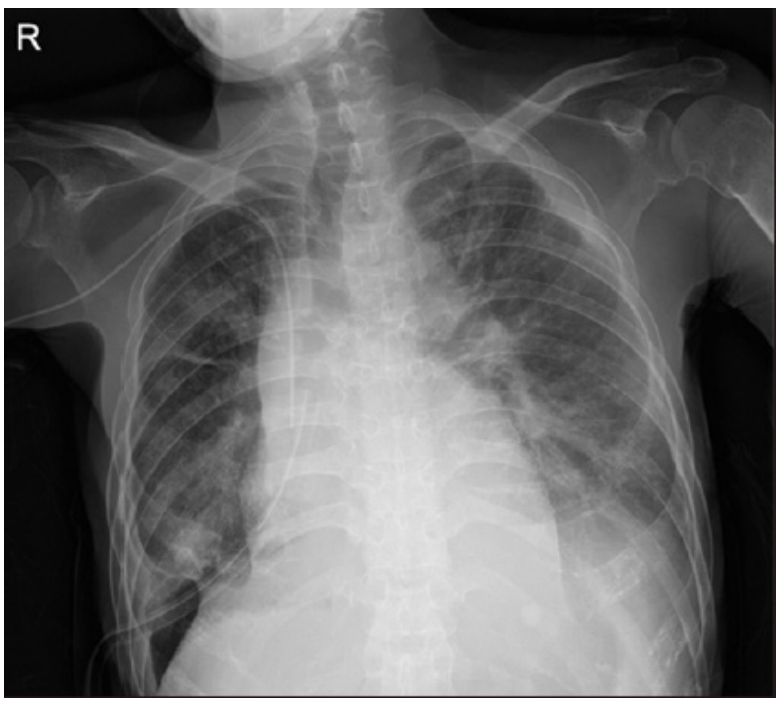

Figure 2. Catheter tip located between the superior vena cava and midportion of the right atrium. guidewire passage failure, and 3) malposition of the catheter tip to another site. Major malposition refers to a catheter tip located in the internal jugular (Fig. 3A) or subclavian vein (Fig. 3B). Minor malposition refers to a catheter tip 1 to $2 \mathrm{~cm}$ above the SVC (Fig. 3C) or deep portion of the RA (Fig. 3D).

First-puncture success refers to one-site puncture without interruption. Multiple-puncture success refers to insert PICC but with an additional puncture site on the ipsilateral or contralateral limb. Shock was diagnosed when the systolic arterial blood pressure was less than $90 \mathrm{mmHg}$ or a vasopressor was administered. Sepsis was defined according to the new Sepsis-3 definitions [8]. AKI was indicated by an elevation of serum creatinine to 1.5 times greater than baseline or a urine output of less than $0.5 \mathrm{~mL} / \mathrm{kg} / \mathrm{hr}$ for 6 to 12 hours (Kidney Disease: Improving Global Outcomes [KDIGO] 2012 AKI clinical practice guideline) [9]. Baseline 


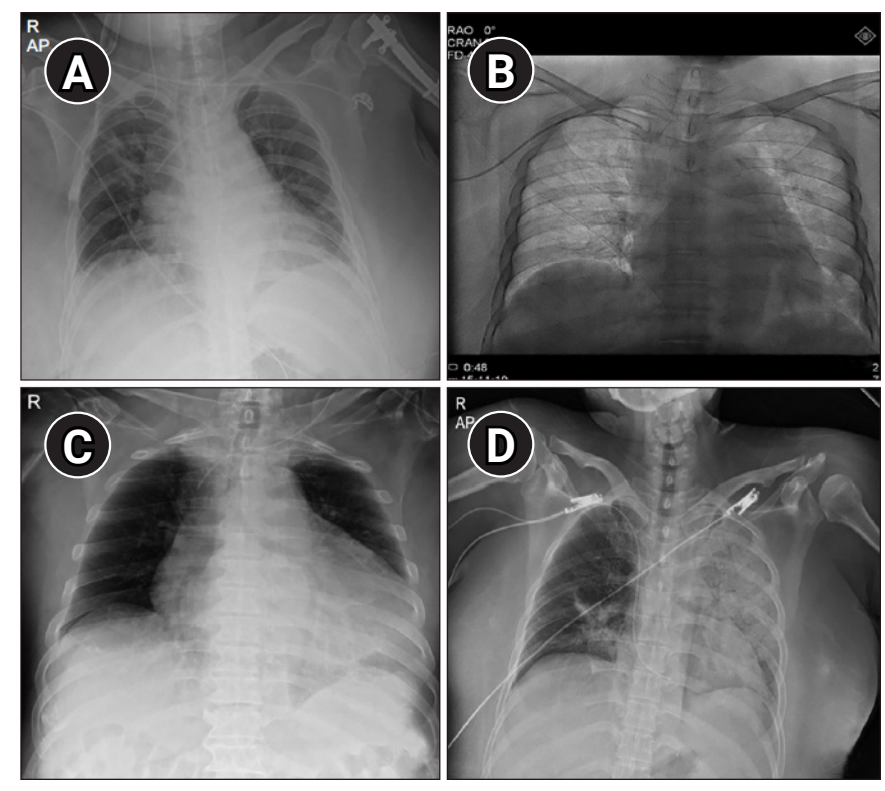

Figure 3. Malposition of catheter tip. (A) Catheter tip located in the internal jugular vein. (B) Catheter tip located in the subclavian vein. (C) Catheter tip located 1 to $2 \mathrm{~cm}$ above the superior vena cava. (D) Catheter tip located in the deep portion of the right atrium.

serum creatinine and eGFR values were from laboratory findings of a previous stable period or periods after recovery from AKI.

\section{Statistical analysis}

Data were analyzed using SPSS ver. 12.0 program (SPSS Inc., Chicago, IL, USA). A p-value less than 0.05 was considered significant. This study was conducted at Samsung Changwon Hospital, Sungkyunkwan University School of Medicine and was approved by the Institutional Review Board (No. 202007-004).

\section{Results}

\section{Demographic and clinical information}

From January 2019 to June 2020, 224 PICC insertions were performed by one nephrologist. Group 1 patients $(n=98)$ had PICCs inserted using an ultrasound-guided method in the ICU (89.8\%) or ward treatment rooms (10.2\%). Group 2 patients $(\mathrm{n}=126)$ had PICCs inserted under fluoroscopic guidance in the angiography room (100\%).
Demographic and clinical information were compared between groups (Table 1). Mean age (71.26 \pm 13.30 vs. $75.32 \pm 11.47$ years, $\mathrm{p}=0.007$ ) was lower in group 1 patients than group 2 patients. Sex ratio (female, $43.9 \%$ vs. $51.6 \%$, $\mathrm{p}=0.282$ ), implementation site (right, $40.8 \%$ vs. $53.2 \%$, $\mathrm{p}$ $=0.080$ ), access vein (basilic vein/brachial vein $/$ cephalic vein, $54.1 \% / 42.9 \% / 3.0 \%$ vs. $54.8 \% / 40.4 \% / 4.8 \%$, $\mathrm{p}=0.788)$, prevalence of diabetes mellitus ( $43.9 \%$ vs. $34.9 \%, p=0.345$ ), and prevalence of hypertension ( $38.8 \%$ vs. $47.6 \%, \mathrm{p}=0.365$ ) were not significantly different between groups. Serum creatinine ( $1.56 \pm 1.45$ vs. $1.52 \pm 1.01 \mathrm{mg} / \mathrm{dL}, \mathrm{p}=0.825)$ and eGFR $\left(72.32 \pm 43.47\right.$ vs. $57.57 \pm 34.13 \mathrm{~mL} / \mathrm{min} / 1.73 \mathrm{~m}^{2}, \mathrm{p}=$ 0.094 ) levels at the time of a stable condition were similar in the two groups. Serum creatinine $(2.24 \pm 2.02$ vs. $2.31 \pm$ $1.94 \mathrm{mg} / \mathrm{dL}, \mathrm{p}=0.788)$, eGFR ( $60.81 \pm 50.71$ vs. $56.83 \pm 62.88$ $\mathrm{mL} / \mathrm{min} / 1.73 \mathrm{~m}^{2}, \mathrm{p}=0.610$ ), hemoglobin (9.67 $\pm 1.40 \mathrm{vs}$. $10.42 \pm 1.82 \mathrm{~g} / \mathrm{dL}, \mathrm{p}=0.128)$, platelet count (128.75 \pm 75.00 vs. $\left.221.80 \pm 64.50 \times 10^{3} / \mathrm{mm}^{3}, \mathrm{p}=0.148\right)$, prothrombin time ( $15.26 \pm 6.50$ vs. $15.30 \pm 6.66$ seconds, $p=0.993$ ), activated partial thromboplastin time ( $33.84 \pm 6.19$ vs. $35.53 \pm 8.03$ seconds, $\mathrm{p}=0.715)$, and serum albumin level ( $2.78 \pm 0.70$ vs. $2.82 \pm 0.66 \mathrm{~g} / \mathrm{dL}, \mathrm{p}=0.993)$ at the time of catheter insertion were also not different between the two groups. Underlying comorbidities were more common in group 1 than group 2 . Sepsis (62.2\% vs. $30.2 \%, \mathrm{p}<0.001)$, shock (52.0\% vs. $4.8 \%, \mathrm{p}<$ $0.001)$, AKI ( $70.4 \%$ vs. $47.6 \%, \mathrm{p}=0.001)$, ventilator use $(62.2 \%$ vs. $0.0 \%, \mathrm{p}<0.001)$, and postoperative state rates $(8.2 \%$ vs. $0.8 \%, \mathrm{p}=0.011$ ) were more common in group 1 than group 2. In-hospital mortality ( $44.9 \%$ vs. $20.6 \%, \mathrm{p}<0.001$ ) was also higher in group 1 than group 2.

\section{Success and complication rates}

Success and complication rates were compared between groups (Table 2). Median duration (IQR) of PICC catheter insertion ( 26 days [ $10-35$ days] vs. 20 days [15-30 days], $p$ $=0.597)$. was not different between the groups. Insertion success rate was high, with no significant difference between the two groups ( $93.9 \%$ vs. $97.6 \%, p=0.171)$. In group 1 , a total of six insertions were failures. No puncture failure cases occurred. Two cases failed due to guidewire passage disturbance, and a PICC was successfully inserted in one of these cases by changing to the fluoroscopic method. The cause of guidewire passage disturbance was the angle of the cephalic arch that blocked smooth passage of the 
Table 1. Patient characteristics $(n=224)$

\begin{tabular}{|c|c|c|c|}
\hline Variable & Group $1(n=98)$ & Group $2(n=126)$ & $\mathrm{p}$-value \\
\hline Age (yr) & $71.26 \pm 13.30$ & $75.32 \pm 11.47$ & 0.007 \\
\hline Female sex & 43 (43.9) & $65(51.6)$ & 0.282 \\
\hline \multicolumn{4}{|l|}{ Comorbidity } \\
\hline Diabetes mellitus & 43 (43.9) & $44(34.9)$ & 0.345 \\
\hline Hypertension & $38(38.8)$ & $60(47.6)$ & 0.365 \\
\hline ICU length of stay (day) & $88(89.8)$ & $12(9.5)$ & $<0.001$ \\
\hline Sepsis & $61(62.2)$ & $38(30.2)$ & $<0.001$ \\
\hline Shock & $51(52.0)$ & $6(4.8)$ & $<0.001$ \\
\hline Acute kidney injury & $69(70.4)$ & $60(47.6)$ & 0.001 \\
\hline Ventilator use & $61(62.2)$ & $0(0)$ & $<0.001$ \\
\hline Congestive heart failure & $12(12.2)$ & $7(5.6)$ & 0.092 \\
\hline Postoperation & $8(8.2)$ & $1(0.8)$ & 0.011 \\
\hline Gastrointestinal bleeding & $2(2.0)$ & $0(0)$ & 0.190 \\
\hline Mortality & $44(44.9)$ & $26(20.6)$ & $<0.001$ \\
\hline \multicolumn{4}{|l|}{ Baseline laboratory finding } \\
\hline Serum creatinine $(\mathrm{mg} / \mathrm{dL})$ & $1.56 \pm 1.45$ & $1.52 \pm 1.01$ & 0.825 \\
\hline eGFR $\left(\mathrm{mL} / \mathrm{min} / 1.73 \mathrm{~m}^{2}\right)$ & $72.32 \pm 43.47$ & $57.57 \pm 34.13$ & 0.094 \\
\hline \multicolumn{4}{|l|}{ Laboratory finding at catheter insertion } \\
\hline Serum creatinine (mg/dL) & $2.24 \pm 2.02$ & $2.31 \pm 1.94$ & 0.788 \\
\hline eGFR $\left(\mathrm{mL} / \mathrm{min} / 1.73 \mathrm{~m}^{2}\right)$ & $60.81 \pm 50.71$ & $56.83 \pm 62.88$ & 0.610 \\
\hline Hemoglobin (g/dL) & $9.67 \pm 1.40$ & $10.42 \pm 1.82$ & 0.128 \\
\hline Platelet $\left(\times 10^{3} / \mathrm{mm}^{3}\right)$ & $128.75 \pm 75.00$ & $221.80 \pm 64.50$ & 0.148 \\
\hline Prothrombin time (sec) & $15.26 \pm 6.50$ & $15.30 \pm 6.66$ & 0.993 \\
\hline Activated partial thromboplastin time (sec) & $33.84 \pm 6.19$ & $35.53 \pm 8.03$ & 0.715 \\
\hline Albumin (g/dL) & $2.78 \pm 0.70$ & $2.82 \pm 0.66$ & 0.993 \\
\hline \multicolumn{4}{|l|}{ Information on catheter insertion } \\
\hline Right side Insertion & $40(40.8)$ & $67(53.2)$ & 0.080 \\
\hline Access vein & & & 0.788 \\
\hline Basilic vein & $53(54.1)$ & $69(54.8)$ & \\
\hline Brachial vein & $42(42.9)$ & $51(40.4)$ & \\
\hline Cephalic vein & $3(3.0)$ & $6(4.8)$ & \\
\hline
\end{tabular}

Data are presented as mean \pm standard deviation or number (\%). eGFR, estimated glomerular filtration rate; ICU, intensive care unit.

guidewire, as detected by venogram (Supplementary Fig. 4, available online). The other example of guidewire passage disturbance occurred after successful puncture; the method was changed to fluoroscopy but failed due to multiple venous stenoses. Major malposition, wherein the catheter tip was placed in the right jugular vein, occurred in one case. Three minor malpositions occurred in group 1. In two cases, the catheter tip was located in an innominate vein above the SVC; in the third case, the catheter tip was located at the deep RA. For these minor malpositions, catheter function was good and the catheter was used without exchange. Malpositions developed in three among 42 brachial vein access cases, one of 53 basilic vein access cases, and none of three cephalic vein access cases in group 1 .

Three cases in group 2 failed. No puncture failures occurred. In one case, guidewire passage disturbance occurred due to venous stenosis. There were two major malposition cases of central vein stenosis where the catheter tip was located in the subclavian vein (Fig. 3B). There were no minor malpositions in group 2 because catheter length measurements were stricter. In group 2, all malposition cases were confirmed to have central vein stenosis.

Multiple-puncture rates among successful cases $(4.1 \%$ vs. $0.0 \%, \mathrm{p}=0.035$ ) were higher in group 1 than group 2 . 
Table 2. Results of PICC procedures between the two groups

\begin{tabular}{|c|c|c|c|}
\hline Variable & Group $1(n=98)$ & Group $2(n=126)$ & p-value \\
\hline Duration of PICC insertion (day) & $26(10-35)$ & $20(15-30)$ & 0.597 \\
\hline Successful cases ${ }^{a}$ & $92(93.9)$ & $123(97.6)$ & 0.171 \\
\hline Failure cases $^{b}$ & $6(6.1)$ & $3(2.4)$ & 0.216 \\
\hline \multicolumn{4}{|l|}{ Cause of failure } \\
\hline Puncture failure & $0(0)$ & $0(0)$ & \\
\hline Guidewire passage & $2(2.0)$ & $1(0.8)$ & $>0.999$ \\
\hline Malposition & $4(4.1)$ & $2(1.6)$ & 0.505 \\
\hline Major malposition ${ }^{c}$ & $1(1.0)$ & $2(1.6)$ & 0.568 \\
\hline Minor malposition $^{d}$ & $3(3.1)$ & $0(0)$ & \\
\hline Multiple puncture rate in successful cases & $4(4.1)$ & $0(0)$ & 0.035 \\
\hline \multicolumn{4}{|l|}{ Insertion-associated complications } \\
\hline Bleeding & $5(5.1)$ & $8(6.3)$ & 0.779 \\
\hline Thrombosis & $1(1.0)$ & $2(1.6)$ & 0.714 \\
\hline Arrhythmia & $0(0)$ & $0(0)$ & \\
\hline Exit site cellulitis & $2(2.0)$ & $1(0.8)$ & 0.582 \\
\hline Systemic infection & $2(2.0)$ & $4(3.2)$ & 0.698 \\
\hline
\end{tabular}

Data are presented as median (interquartile range) or number (\%).

PICC, peripherally inserted central catheter.

${ }^{a}$ Good catheter function with the catheter tip located between the superior vena cava and mid-portion of the right atrium. ${ }^{\mathrm{b} S u b s t a n d a r d ~ i n f l o w / o u t f l o w ~ o r ~}$

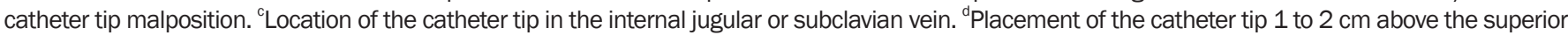
vena cava or deep portion of the right atrium.

Insertion site bleeding $(5.1 \%$ vs. $6.3 \%, \mathrm{p}=0.779)$ was the most common complication in both groups. Thrombosis incidence ( $1.0 \%$ vs. $1.6 \%, \mathrm{p}=0.714)$ was low and was not different between the two groups. Insertion site exit infections ( $2.0 \%$ vs. $0.8 \%, \mathrm{p}=0.582)$ and systemic infections $(2.0 \%$ vs. $3.2 \%, \mathrm{p}=0.698)$ were rare in both groups.

\section{Discussion}

An increasing number of elderly patients and those who have hemodynamic instability, are in a state of shock, or are ventilator-dependent are admitted to the nephrology department or receive consultations from this department [3]. These patients need external support such as proper intravenous therapy due to long-term hospitalization, requirement for intravenous antibiotics, and nutritional therapy. However, lack of blood vessel access in this patient population can hinder proper treatment. To treat these patients in the ICU, a central catheter needs to be inserted. Considering the higher incidence of CKD development in AKI patients, a centrally inserted central catheter (CICC) may be better in patients with AKI as indicated by a serum creatinine level greater than 2.0 or $3.0 \mathrm{mg} / \mathrm{dL}$ for vascular protection. However, if clinical necessity is considered more significant than future access creation, a PICC may be a useful alternative. Traditionally, a CICC is inserted through a subclavian vein or the internal jugular vein. In recent years, PICCs have increasingly been used in critical care settings because of their benefits over CICCs [10]. First, their insertion is easy and safe, simply involving puncture and cannulation of a peripheral vein of the arm. PICC insertion and United States guidance negate the risk of hemothorax and pneumothorax, and the possibility of primary malposition is very low $[11,12]$. Furthermore, PICC placement is appropriate in patients with coagulative disorders who need central vein access to avoid post-procedural hemorrhage [11,13]. PICCs also have a low catheter-related blood flow infection rate [14]. At present, PICCs are highly recommended in the following clinical conditions; major anatomic abnormalities of the chest and neck that may lead to difficulties in the placement and dressing of CICCs, tracheostomy, and decreased platelet count or coagulation abnormalities [11]. PICC use has also been recommended in critically ill patients with severe cardiopulmonary problems, severe malnutrition, or obesity [12].

However, the increased use of PICCs has increased the 
incidence of complications such as venous thrombosis and infection. PICC use is acceptable only when the duration of use is scheduled to be longer than 6 days or non-peripherally compatible infusions are needed (e.g., sclerosing antibiotics or chemotherapy) [15]. Among patients with CKD stage 3b or higher (eGFR of $<45 \mathrm{~mL} / \mathrm{min} / 1.73 \mathrm{~m}^{2}$ ), PICC use is generally considered unacceptable, largely due to the high likelihood of peripheral and central venous complications (including thrombosis) interrupting future hemodialysis access [16]. These types of cases require nephrology consultation prior to PICC placement for assessment of risk vs. benefit; PICCs are only strongly argued for in cases of clinical necessity. When venous access is necessary for 5 or fewer days, experts recommend placement of peripheral intravenous catheters in the dorsum of the hand (avoiding the forearm veins) for infusion of peripherally compatible infusates. If venous access is needed for longer durations or for infusion of a non-peripherally compatible drug, the use of tunneled small-bore central catheters (for example, 4-Fr single-lumen or 5-Fr double-lumen catheters inserted in the jugular vein and tunneled toward the chest) is considered appropriate [17]. This study excluded patients with CKD (stage 3b and above) who did not undergo dialysis and patients receiving maintenance dialysis.

A PICC is commonly inserted in the angiography room, but insertion can be conducted at the patient's bedside when risks associated with moving the patient are considered too high $[18,19]$. Nephrologists have accumulated considerable experience in the use of ultrasound for insertion of tunneled catheters; therefore, they can perform ultrasound-guided procedures without problems. In the current study, it was difficult to insert the guidewire or catheter in some cases. In these cases, the cause was stenosis or excessive curvature of the vein. In cases of passage disturbance of the guidewire, a fluoroscopic venogram can be performed for guidewire rerouting or an alternate puncture site can be used. In ultrasound-guided bedside procedures, this can be conducted by selecting a different puncture site or using a more proximal vein.

In particular, depending on the angle at which the cephalic arch meets the subclavian vein, guidewire passage is sometimes difficult. The basilic vein is preferred as it is the largest diameter upper extremity vessel and affords a nontortuous entry route into the subclavian vein. The cephalic vein is smaller than the basilic vein and makes a $90^{\circ}$ angle at its entry to the terminal portion of the axillary vein, making catheter advancement somewhat difficult. Brachial veins lie deep in the center of the mid to upper arm and cannot be outwardly visualized or palpated; ultrasound guidance is required for access. In this study, malposition-prone access veins were not definite. First, malposition events were rare. Second, a minor malposition depends on length rather than type of access vein. Of the 11 patients with a dialysis catheter in group 1, six patients had a PICC implanted on the ipsilateral side and five patients had a PICC implanted on the other side. No entry barrier to the guidewire was found during insertion in either group (data not shown).

Selecting the exact location of the PICC at the bedside is difficult without fluoroscopic images. This can lead to possible position abnormalities. In existing studies, the probability of location abnormality was variously reported to be $3 \%$ to $37 \%$ [20-23], $8.5 \%$ [4], and $7.9 \%$ [5]. However, there is no widespread agreement between experts regarding the correct position for the tip of a PICC [24-26]. Most American recommendations (Association for Vascular Access, Food and Drug Administration) $[27,28]$ suggest that the tip be in the inferior third of the SVC, while European guidelines $[11,29]$ recommend positioning of the tip in the RA (specifically, in the upper area) appropriate. A widely accepted opinion is that the optimal site is proximal to the area between the SVC and RA [30]. If the catheter tip is in a higher position (i.e., in the brachiocephalic, internal jugular, or subclavian vein), there is an increased risk of malfunction [31] and an increased risk of venous thrombosis compared to a position lower in the SVC or close to the cavoatrial junction [32]. If the tip is positioned "too low" (RA, right ventricle, or inferior vena cava), there is a risk of arrhythmia, tricuspid valve dysfunction or lesions, and thrombosis [33,34].

In addition, PICC removal and reinsertion due to abnormal location may be difficult, particularly if blood vessel condition is poor. In this study, catheters malpositioned near the SVC or RA had good function and long-term patency. Position abnormality corrections can be accomplished by assessing the internal jugular veins with ultrasound during insertion. The absence (Supplementary Fig. 2) or presence (Supplementary Fig. 3) of the guidewire can be easily checked with ultrasound, and, if visible, the guidewire can be moved back to the heart through shaping of the catheter tip. This can reduce the incidence of positional abnormality on chest radiograph after the procedure from $7.4 \%$ to $0.7 \%$ [35]. 
Infection (cellulitis, abscesses, or bacteremia and systemic infections), phlebitis/infiltration, mechanical malfunction, air embolism, cardiac arrhythmias, and catheter occlusions are the main complications associated with PICC insertion [7]. In this study, the most common complications were exit site bleeding that was controlled by compression dressing. One patient (Supplementary Fig. 5, available online) went into cardiogenic shock because of acute massive myocardial infarction. Low blood pressure may lead to the need to apply ECMO, and subsequent AKI can lead to CRRT using the ECMO line. These scenarios are common in modern ICU AKI patients. These patient require a PICC line for longterm hospitalization. In the current study, two patients had successful PICC insertion without bleeding complications despite persistent heparin anticoagulation. Furthermore, thrombosis and infection were rare, neither group had serious complications, and no mortalities occurred in either group.

This study has some limitations that should be considered. First, because insertions were performed by one practitioner at a single center, the results cannot be generalized to other nephrologists. Second, only short-term in-hospital clinical outcomes during admission were analyzed. Long-term outcomes and patient survival taking into account recovery from AKI and central venous stenosis should be assessed in future research.

In conclusion, PICC insertion by a nephrologist was highly successful and safe when performed in critically ill patients with comorbidities. Furthermore, the success rate of bedside PICC insertion was comparable to that of PICC insertion under fluoroscopic guidance. Adequate placement of the appropriate catheter is important in the management of critically ill patients to improve their prognosis and reduce avoidable complications. PICC insertion is generally contraindicated in CKD patients for arm-save, but in a life-threatening situation in the ICU, PICC insertion may be considered even in CKD patients, and under these circumstances, PICCs can be safely placed by nephrologists. It is expected that the role of interventional nephrologists in the insertion and management of central catheters will only increase over time.

\section{Conflicts of interest}

The author has no conflicts of interest to declare.

\section{ORCID}

Seong Cho, https://orcid.org/0000-0002-6183-1647

\section{References}

1. Lam S, Scannell R, Roessler D, Smith MA. Peripherally inserted central catheters in an acute-care hospital. Arch Intern Med 1994;154:18331837.

2. Ng PK, Ault MJ, Ellrodt AG, Maldonado L. Peripherally inserted central catheters in general medicine. Mayo Clin Proc 1997;72:225233.

3. Alston H, Burns A. Ageing renal patients: we need more collaboration between geriatric services and nephrology departments. Healthcare (Basel) 2015;3:1075-1085.

4. Lee DS, Park CM. Clinical feasibility of ultrasound guided placement of peripherally inserted central catheters by intensivist: preliminary report. J Acute Care Surg 2014;4:13-17.

5. Song L, Li H. Malposition of peripherally inserted central catheter: experience from 3,012 patients with cancer. Exp Ther Med 2013;6:891-893.

6. Kim HJ, Jung CY, Bae JM. Clinical characteristics of peripherally inserted central catheter in critically ill patients. J Acute Care Surg 2019;9:18-24.

7. Gonzalez R, Cassaro S. Percutaneous central catheter. In: Abai B, Abu-Ghosh A, Acharya AB, editors. StatPearls. Treasure Island (FL): StatPearls Publishing; 2020.

8. Shankar-Hari M, Phillips GS, Levy ML, et al. Developing a new definition and assessing new clinical criteria for septic shock: for the third International Consensus Definitions for Sepsis and Septic Shock (Sepsis-3). JAMA 2016;315:775-787.

9. Kidney Disease: Improving Global Outcomes Acute Kidney Injury Work Group. KDIGO clinical practice guideline for acute kidney injury. Kidney Int Suppl 2012;2:1-138.

10. Griffiths VR, Philpot P. Peripherally inserted central catheters (PICCs): do they have a role in the care of the critically ill patient? Intensive Crit Care Nurs 2002;18:37-47.

11. Pittiruti M, Hamilton H, Biffi R, MacFie J, Pertkiewicz M; ESPEN. ESPEN guidelines on parenteral nutrition: central venous catheters (access, care, diagnosis and therapy of complications). Clin Nutr 2009;28:365-377.

12. Pittiruti M, Brutti A, Celentano D, et al. Clinical experience with power-injectable PICCs in intensive care patients. Crit Care 2012;16:R21.

13. Gallieni M, Pittiruti M, Biffi R. Vascular access in oncology 
patients. CA Cancer J Clin 2008;58:323-346.

14. Gunst M, Matsushima K, Vanek S, Gunst R, Shafi S, Frankel H. Peripherally inserted central catheters may lower the incidence of catheter-related blood stream infections in patients in surgical intensive care units. Surg Infect (Larchmt) 2011;12:279-282.

15. Chopra V, Flanders SA, Saint S, et al. The Michigan Appropriateness Guide for Intravenous Catheters (MAGIC): results from a multispecialty panel using the RAND/UCLA appropriateness method. Ann Intern Med 2015;163(6 Suppl):S1-S40.

16. El Ters M, Schears GJ, Taler SJ, et al. Association between prior peripherally inserted central catheters and lack of functioning arteriovenous fistulas: a case-control study in hemodialysis patients. Am J Kidney Dis 2012;60:601-608.

17. Sasadeusz KJ, Trerotola SO, Shah H, et al. Tunneled jugular small-bore central catheters as an alternative to peripherally inserted central catheters for intermediate-term venous access in patients with hemodialysis and chronic renal insufficiency. Radiology 1999;213:303-306.

18. Schwebel C, Clec'h C, Magne S, et al. Safety of intrahospital transport in ventilated critically ill patients: a multicenter cohort study. Crit Care Med 2013;41:1919-1928.

19. Parmentier-Decrucq E, Poissy J, Favory R, et al. Adverse events during intrahospital transport of critically ill patients: incidence and risk factors. Ann Intensive Care 2013;3:10.

20. Johnston AO, Clark RG. Malpositioning of central venous catheters. Lancet 1972;2:1395-1397.

21. Lum PS, Soski M. Management of malpositioned central venous catheters. J Intraven Nurs 1989;12:356-365.

22. Fricke BL, Racadio JM, Duckworth T, Donnelly LF, Tamer RM, Johnson ND. Placement of peripherally inserted central catheters without fluoroscopy in children: initial catheter tip position. Radiology 2005;234:887-892.

23. Trerotola SO, Thompson S, Chittams J, Vierregger KS. Analysis of tip malposition and correction in peripherally inserted central catheters placed at bedside by a dedicated nursing team. $J$ Vasc Interv Radiol 2007;18:513-518.
24. Stonelake PA, Bodenham AR. The carina as a radiological landmark for central venous catheter tip position. Br J Anaesth 2006;96:335340.

25. Cotogni P, Pittiruti M. Focus on peripherally inserted central catheters in critically ill patients. World J Crit Care Med 2014;3:80-94.

26. National Association of Vascular Access Networks. Tip location of peripherally inserted central catheters. J Vasc Access Devices 1998;3:8-10.

27. Scott WL. Complications associated with central venous catheters. A survey. Chest 1988;94:1221-1224.

28. Scott WL. Central venous catheters. An overview of Food and Drug Administration activities. Surg Oncol Clin N Am 1995;4:377-393.

29. Royal College of Nursing. I.V. Therapy forum: standards for infusion therapy [Internet]. London (UK): Royal College of Nursing, 2010 [cited 2010 Jan 30]. Available from: http://www. ren.org.uk.

30. Pittiruti M, La Greca A, Scoppettuolo G. The electrocardiographic method for positioning the tip of central venous catheters. J Vasc Access 2011;12:280-291.

31. Petersen J, Delaney JH, Brakstad MT, Rowbotham RK, Bagley CM Jr. Silicone venous access devices positioned with their tips high in the superior vena cava are more likely to malfunction. $\mathrm{Am} \mathrm{J}$ Surg 1999;178:38-41.

32. Caers J, Fontaine C, Vinh-Hung V, et al. Catheter tip position as a risk factor for thrombosis associated with the use of subcutaneous infusion ports. Support Care Cancer 2005;13:325-331.

33. Taylor RW, Palagiri AV. Central venous catheterization. Crit Care Med 2007;35:1390-1396.

34. Korones DN, Buzzard CJ, Asselin BL, Harris JP. Right atrial thrombi in children with cancer and indwelling catheters. J Pediatr 1996;128:841-846.

35. Schweickert WD, Herlitz J, Pohlman AS, Gehlbach BK, Hall JB, Kress JP. A randomized, controlled trial evaluating postinsertion neck ultrasound in peripherally inserted central catheter procedures. Crit Care Med 2009;37:1217-1221. 